\title{
The intra- and extracellular proteome of Aspergillus niger growing on defined medium with xylose or maltose as carbon substrate
}

\author{
Xin Lu, Jibin Sun, Manfred Nimtz, Josef Wissing, An-Ping Zeng and Ursula Rinas*
}

\begin{abstract}
Background: The filamentous fungus Aspergillus niger is well-known as a producer of primary metabolites and extracellular proteins. For example, glucoamylase is the most efficiently secreted protein of Aspergillus niger, thus the homologous glucoamylase ( $\mathrm{g} / \mathrm{a} A)$ promoter as well as the glaA signal sequence are widely used for heterologous protein production. Xylose is known to strongly repress glaA expression while maltose is a potent inducer of glaA promoter controlled genes. For a more profound understanding of A. niger physiology, a comprehensive analysis of the intra- and extracellular proteome of Aspergillus niger AB1.13 growing on defined medium with xylose or maltose as carbon substrate was carried out using 2-D gel electrophoresis/Maldi-ToF and nano-HPLC MS/MS.
\end{abstract}

Results: The intracellular proteome of A. niger growing either on xylose or maltose in well-aerated controlled bioreactor cultures revealed striking similarities. In both cultures the most abundant intracellular protein was the TCA cycle enzyme malate-dehydrogenase. Moreover, the glycolytic enzymes fructose-bis-phosphate aldolase and glyceraldehyde-3-phosphate-dehydrogenase and the flavohemoglobin FhbA were identified as major proteins in both cultures. On the other hand, enzymes involved in the removal of reactive oxygen species, such as superoxide dismutase and peroxiredoxin, were present at elevated levels in the culture growing on maltose but only in minor amounts in the xylose culture. The composition of the extracellular proteome differed considerably depending on the carbon substrate. In the secretome of the xylose-grown culture, a variety of plant cell wall degrading enzymes were identified, mostly under the control of the xylanolytic transcriptional activator XInR, with xylanase B and ferulic acid esterase as the most abundant ones. The secretome of the maltose-grown culture did not contain xylanolytic enzymes, instead high levels of catalases were found and glucoamylase (multiple spots) was identified as the most abundant extracellular protein. Surprisingly, the intracellular proteome of A. niger growing on xylose in bioreactor cultures differed more from a culture growing in shake flasks using the same medium than from the bioreactor culture growing on maltose. For example, in shake flask cultures with xylose as carbon source the most abundant intracellular proteins were not the glycolytic and the TCA cycle enzymes and the flavohemoglobin, but CipC, a protein of yet unknown function, superoxide dismutase and an NADPH dependent aldehyde reductase. Moreover, vacuolar proteases accumulated to higher and ER-resident chaperones and foldases to lower levels in shake flask compared to the bioreactor cultures.

Conclusions: The utilization of xylose or maltose was strongly affecting the composition of the secretome but of minor influence on the composition of the intracellular proteome. On the other hand, differences in culture conditions ( $\mathrm{pH}$ control versus no $\mathrm{pH}$ control, aeration versus no aeration and stirring versus shaking) have a profound effect on the intracellular proteome. For example, lower levels of ER-resident chaperones and foldases and higher levels of vacuolar proteases render shake flask conditions less favorable for protein production compared to controlled bioreactor cultures.

(c) 2010 Lu et al; licensee BioMed Central Ltd. This is an Open Access article distributed under the terms of the Creative Commons AttriB.Wed Central bution License (http://creativecommons.org/licenses/by/2.0), which permits unrestricted use, distribution, and reproduction in any medium, provided the original work is properly cited. 


\section{Background}

The filamentous fungus Aspergillus niger is well-known as a producer of primary metabolites and extracellular enzymes. For example, there are reports that glucoamylase, a starch degrading enzyme used in the food industry, is produced as extracellular protein in 20 grams per liter quantities [1]. Because glucoamylase is the most efficiently secreted protein of Aspergillus niger, the homologous glucoamylase $(g l a A)$ promoter as well as the glucoamylase signal sequence are widely used for heterologous protein production. This way the recombinant protein can be directed towards the culture medium facilitating further down-stream processing. However, limitations in the secretory pathway and proteolytic degradation often hamper the extracellular accumulation of the recombinant product. Also, regulation of glaA promoter controlled protein production is not fully understood, though it is clear that induction occurs when the growth medium contains starch or its degradation products maltodextrine, maltose or glucose and repression is observed when growth occurs on xylose as carbon source [2-9]. Thus, induction of glaA promoter controlled protein production is most often carried out by maltose addition to cultures pregrown on xylose $[8,10]$.

Little is known about the composition of the proteome of Aspergilli. However, the sequenced genomes of some Aspergilli are now publicly available, including also the first annotated genomic sequence of $A$. niger [11] fostering now strain improvement based on integrated genomics [12]. Although annotation is far from being complete, protein identification based on peptide-mass fingerprinting is starting to become routine also for $A$. niger. Recent studies on $A$. niger proteomics are limited either to the intra- or to the extracellular proteome. A comparative study on the extracellular proteome of $A$. niger growing on different substrates has been carried out using LC/MS [13]. Moreover, the intracellular proteome of $A$. niger was analyzed under conditions leading to enhanced production of the mycotoxin fumonisin using 2-D electrophoresis and Maldi-ToF [14].

In this work, we performed a comprehensive analysis of the intra- as well as the extracellular proteome of $A$. niger growing under different culture conditions. Cultivations were carried out in well-aerated controlled bioreactor cultures as well as in shake flask culture using a defined medium with xylose or maltose as carbon source. Moreover, the time-dependent change of the extracellular pro-

\footnotetext{
* Correspondence: Ursula.Rinas@helmholtz-hzi.de

1 Helmholtz Center for Infection Research, Inhoffenstr, 7, 38124 Braunschweig, Germany
}

Full list of author information is available at the end of the article teome was also followed in response to maltose addition to a culture pregrown on xylose. Proteome analysis including the identification of individual proteins was done by 2-D gel electrophoresis combined with MaldiToF or Q-ToF and, additionally for extracellular proteins, with liquid chromatography followed by mass spectrometry (LC-MS) analysis based on the annotated A. niger genome sequence [11].

\section{Results and Discussion}

\section{Catabolism of xylose and maltose by A. niger}

Xylose and maltose are both sugar carbon substrates which can be used by $A$. niger as sole carbon substrate leading to comparable growth kinetics and final biomass concentrations in controlled bioreactor cultures (Fig. 1), although both sugars are catabolized by different metabolic routes (Fig. 2). Uptake of xylose occurs in the unmodified form followed by intracellular reduction to xylitol through xylose reductase [15]. Xylitol is then converted to L-xylulose and after phosphorylation further degraded in the pentose phosphate pathway [15]. Maltose cleavage into glucose moieties occurs extracellular by glucoamylase as $A$. niger has no functional uptake system for maltose in contrast to many yeast and other filamentous fungi $[16,17]$. Glucose uptake occurs either directly with subsequent catabolic degradation of glucose via the glycolytic and the pentose phosphate pathway [15] or extracellular oxidation of glucose to gluconic acid by glucose oxidase with concomitant generation of hydrogen peroxide can occur as an alternative route of glucose catabolism. Gluconic acid is then further metabolized via the pentose phosphate pathway $[18,19]$.

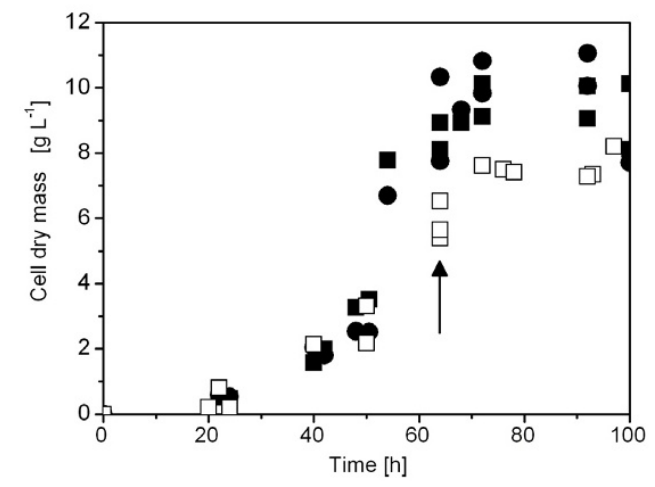

Figure 1 Growth profile of $\boldsymbol{A}$. niger. Growth profile of A. niger AB1.13 in controlled bioreactor (filled symbols) or shake flask cultures (open symbols) using either xylose (squares) or maltose (circles) as carbon substrate. Bioreactor and shake flask cultivations were carried out in duplicate and triplicate, respectively. The arrow points to the sampling point for proteome analysis. 


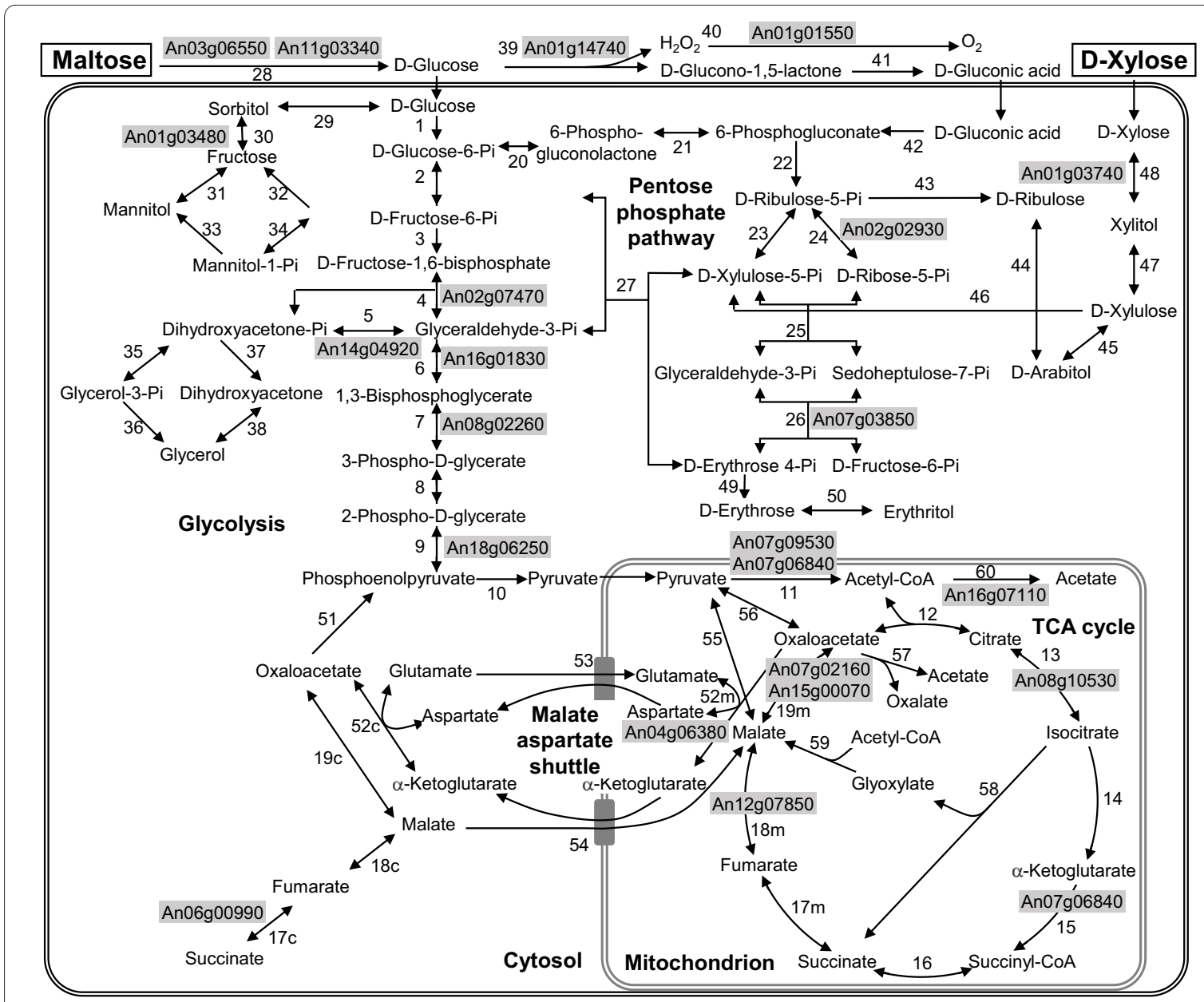

Figure 2 Metabolic network of xylose and maltose catabolism. Simplified scheme of the extra- and intracellular pathways involved in xylose and maltose catabolism in A. niger [64]. The reaction numbers correspond to reactions listed in Additional file 1. Proteins identified from 2-D gels (indicated by gray background) include enzymes from the glycolytic pathway: $\mathbf{4}$ (fructose-bis-phosphate aldolase, An02g07470); $\mathbf{5}$ (triose phosphate isomerase, An14g04920); 6 (glyceraldehde-3-phosphate dehydrogenase, An16g01830); 7 (phosphoglycerate kinase, An08g02260); 9 (enolase, An18g06250); 11 (pyruvate dehydrogenase complex: pyruvate dehydrogenase E1, An07g09530, dihydrolipoamide dehydrogenase E3, An07g06840); TCA cycle enzymes: 13 (aconitase, An08g 10530); 15 (a-ketoglutarate dehydrogenas complex: dihydrolipoamide dehydrogenase E3, An07g06840); 18 m (fumarase, An12g07850); 19 m (malate dehydrogenase, An07g02160, An15g00070); enzymes from the pentose phosphate pathway: 24 (ribose-5-phosphate isomerase, An02g02930); 26 (transaldolase, An07g03850); enzymes from the malate-aspartate shuttle: $\mathbf{5 2} \mathbf{~ m}$ (aspartate aminotransferase,

An04g06380); enzymes involved in anaerobic redox balancing: 17c (cytoplasmic fumarate reductase, An06g00990); enzymes involved in polyol metabolism: 30 (sorbitol dehydrogenase, An01g03480); acetate formation (acetyl-CoA hydrolase, An16g071 10); xylose breakdown: 48 (xylose reductase, An01g03740); and the enzymes involved in the extracellular maltose and glucose breakdown: 28 (glucoamylase An03g06550; a-amylase, An11g03340), 39 (glucose oxidase, An01g14740) and $\mathbf{4 0}$ (catalase, An01g01550).

\section{Comparative intracellular proteome analysis of A. niger grown on xylose or maltose in bioreactor cultures} Intracellular proteins of equal abundance in xylose or maltose grown cultures

Despite the different routes of catabolic breakdown of the two carbon substrates, the intracellular proteome of $A$. niger growing either on xylose or maltose revealed striking similarities (Fig. 3). On both carbon substrates, the glycolytic enzymes fructose-bis-phosphate aldolase (Fba1) and glyceraldehyde-3-phosphate-dehydrogenase (GpdA), and the TCA cycle enzyme malate-dehydrogenase (Mdh1), represent the most prominent components of the intracellular proteome (Fig. 3). Furthermore, the flavohemoglobin FhbA accumulates in both cultures to high quantity. The function of this protein is not yet clear; its expression has been correlated with a hyphal growth phenotype [20] and its enzymatic characterization suggests a role in nitrosative stress protection [21]. Homologs of FhbA in Saccharomyces cerevisiae reveal a complex role in the oxidative stress response but no function in respiration [22,23]. This protein seems to be an important fungal protein as its homolog in $P$. chrysogenum also represents a main component of the intracellular proteome [24]. Another major protein found in both 


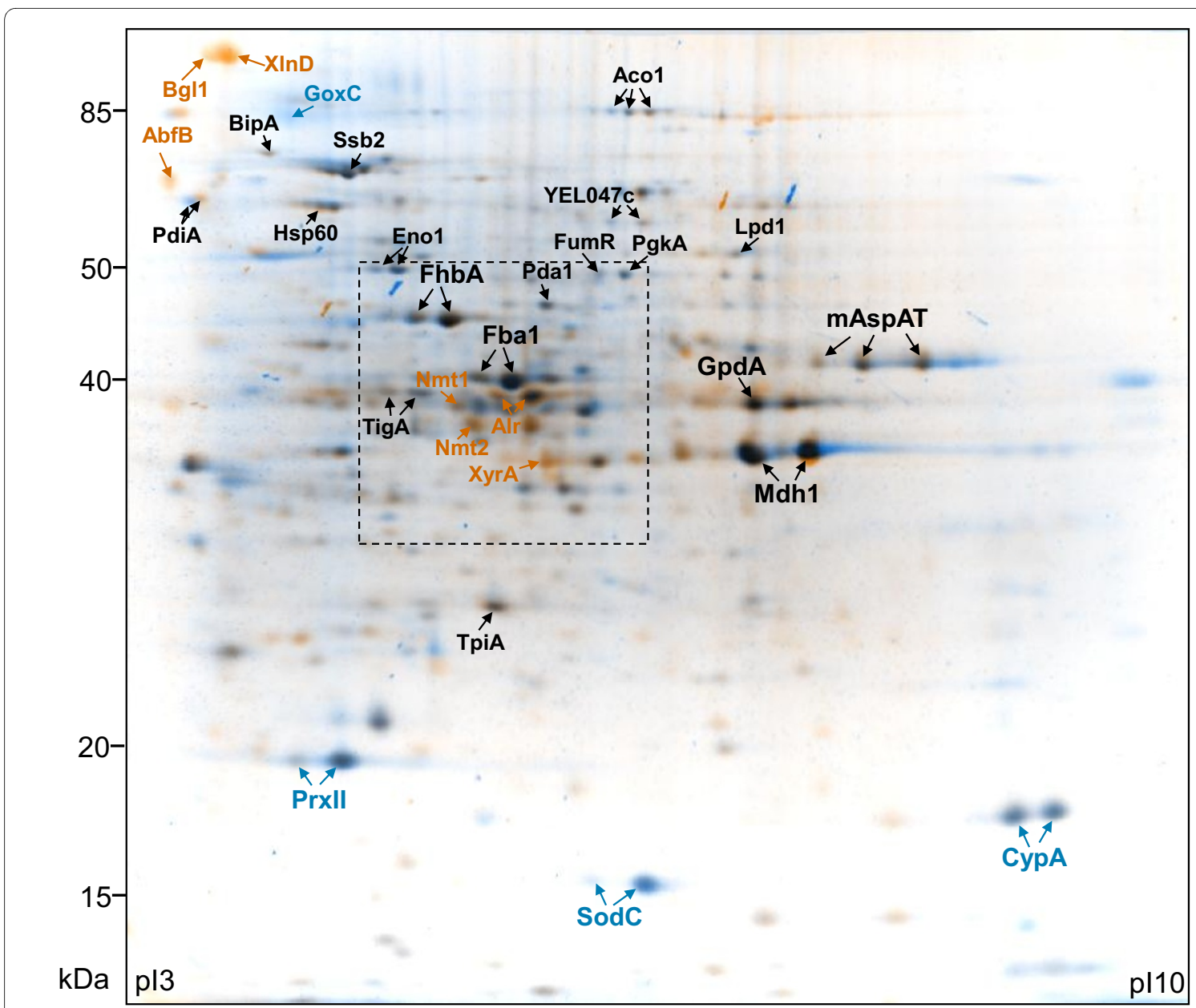

Figure 3 Intracellular proteome of $\boldsymbol{A}$. niger grown on xylose or maltose. Comparative analysis of the intracellular proteome of $A$. niger AB1.13 grown to late exponential/early stationary phase in bioreactor cultures on defined medium with xylose or maltose as carbon substrate. Dual-channel image; orange and blue spots correspond to proteins which are strongly upregulated in the presence of xylose and maltose, respectively. Black spots represent proteins which are found in both cultures. Characteristics of proteins indicated by arrows are discussed in more detail. The orientation of the isoelectric focussing gel is indicated and molecular mass standards are given on the left. The framed insert indicates the section which is shown in more detail in Fig. 5. The 2-D gel images of the intracellular proteome of A. niger AB1.13 from xylose and maltose grown cultures with all identified proteins on interactive and searchable 2-D gels are available as Additional files 3 and 4, respectively. The complete list of proteins from the intracellular proteome of A. niger AB1.13 growing either on xylose or maltose, which have been identified on 2-D gels and classified into functional categories, is found in Additional file 2. A detailed list of all intracellular identified proteins showing significant changes in abundance depending on the carbon substrate is available in Additional file 5.

cultures in comparable abundance is the mitochondrial aspartate aminotransferase (mAspAT), a component of the malate-aspartate shuttle involved in exchanging $\mathrm{NADH}$ formed in the cytosol for mitochondrial NAD ${ }^{+}$.

Other enzymes of the central metabolic pathways, the glycolytic enzymes triose phosphate isomerase (TpiA), phosphoglycerate kinase (PgkA), and phosphopyruvate hydratase or enolase (Eno1), enzymes of the pyruvate dehydrogenase complex, namely the $\alpha$ subunit E1 (Pda1) and the dihydrolipoamide dehydrogenase (Lpd1, also present in the TCA cycle $\alpha$-ketoglutarate dehydrogenase complex), and the other TCA cycle enzymes aconitase (Aco1) and fumarate hydratase or fumarase (FumR) and the cytoplasmic fumarate reductase (YEL047c) are found as minor spots but also of equal abundance in both cultures. Thus, enzymes of the central metabolic pathways accumulate to similar abundance in both cultures growing either on maltose or xylose as carbon source. Enzymes from central metabolic pathways identified on 2-D gels are indicated in Fig. 2. A complete list of metabolic reactions and corresponding enzymes identified on 2-D gels is found in Additional file 1.

Other important proteins present in similar amounts in the mycelium of $A$. niger grown either on xylose or maltose are ER-resident chaperones and foldases of the secretory pathway such as the DnaK-type molecular 
chaperones BipA and Ssb2, protein disulfide isomerases PdiA and TigA as well as heat shock proteins such as Hsp60 indicating that the maltose-induced synthesis of the extracellular glucoamylase has no influence on the amounts of ER-resident chaperones and foldases. A complete list of proteins from the intracellular proteome of $A$. niger AB1.13 grown either on xylose or maltose, which have been identified on 2-D gels and classified into functional categories, is found in the Additional file 2. Moreover, interactive and searchable 2-D gels of the intracellular proteome of $A$. niger $\mathrm{AB} 1.13$ from the xylose and maltose grown culture with all identified proteins indicated in Additional file 2 are found in Additional files 3 and 4 , respectively.

\section{Intracellular proteins found in higher abundance in the xylose-grown culture}

Proteins only present during growth on xylose are Dxylose reductase $(\mathrm{XyrA}), \beta$-xylosidase $(\mathrm{X} \ln \mathrm{D})$, arabinofuranosidase $B(A b f B)$ and $\beta$-glucosidase (Bgl1 or BglA) (Fig. 3, Additional file 5). XyrA catalyses the NADPH dependent reduction of xylose to xylitol, which represents the first step in D-xylose metabolism. Expression of the $x y r A$ gene is under the control of the xylanolytic transcriptional activator $X \ln R[25,26]$. XlnD, also under control of XlnR, catalyses the hydrolysis of xylooligosaccharides [26,27]. It is also present in the medium (Fig. 4), and, thus probably as cell wall associated protein present in the mycelium. AbfB and Bgl1, both not con- trolled by $X \operatorname{lnR}$ [28], are enzymes involved in the hydrolytic release of $\mathrm{L}$-arabinofuranosyl residues from arabinoxylan and arabinan and in glucose moieties from gluco-oligosaccharides, respectively. Both enzymes are also found in the medium, and, thus, are probably as $\mathrm{X} \ln \mathrm{D}$ present as cell wall associated proteins.

Some other proteins are not exclusive to the xylose grown culture but accumulate to significantly higher amounts compared to the maltose grown culture. Noticeable examples are an NADPH dependent aldehyde reductase (Alr), and enzymes involved in the biosynthesis of thiamine, namely Nmt1 and Nmt2. The physiological function of the NADPH dependent aldehyde reductase (Alr) is yet unknown, as member of the aldo-keto reductase superfamily it may perform complex roles in carbohydrate and detoxification pathways [29,30]. Nmt1 is involved in the synthesis of thiamine, being responsible for the synthesis of the pyrimidine moiety of thiamine (vitamin B1) [31]. Nmt2 plays a central role in the biosynthesis of thiazole, a precursor of thiamine [32]. In fission yeast, it has been shown that both genes are co-ordinately controlled, highly transcribed in minimal medium and completely repressed in the presence of thiamine [32]. There are also indications that Nmt2 (Thi4 in S. cerevisiae) may have some role in providing oxidative damage tolerance [33,34]. A detailed list of all identified intracellular proteins showing significant changes in abundance
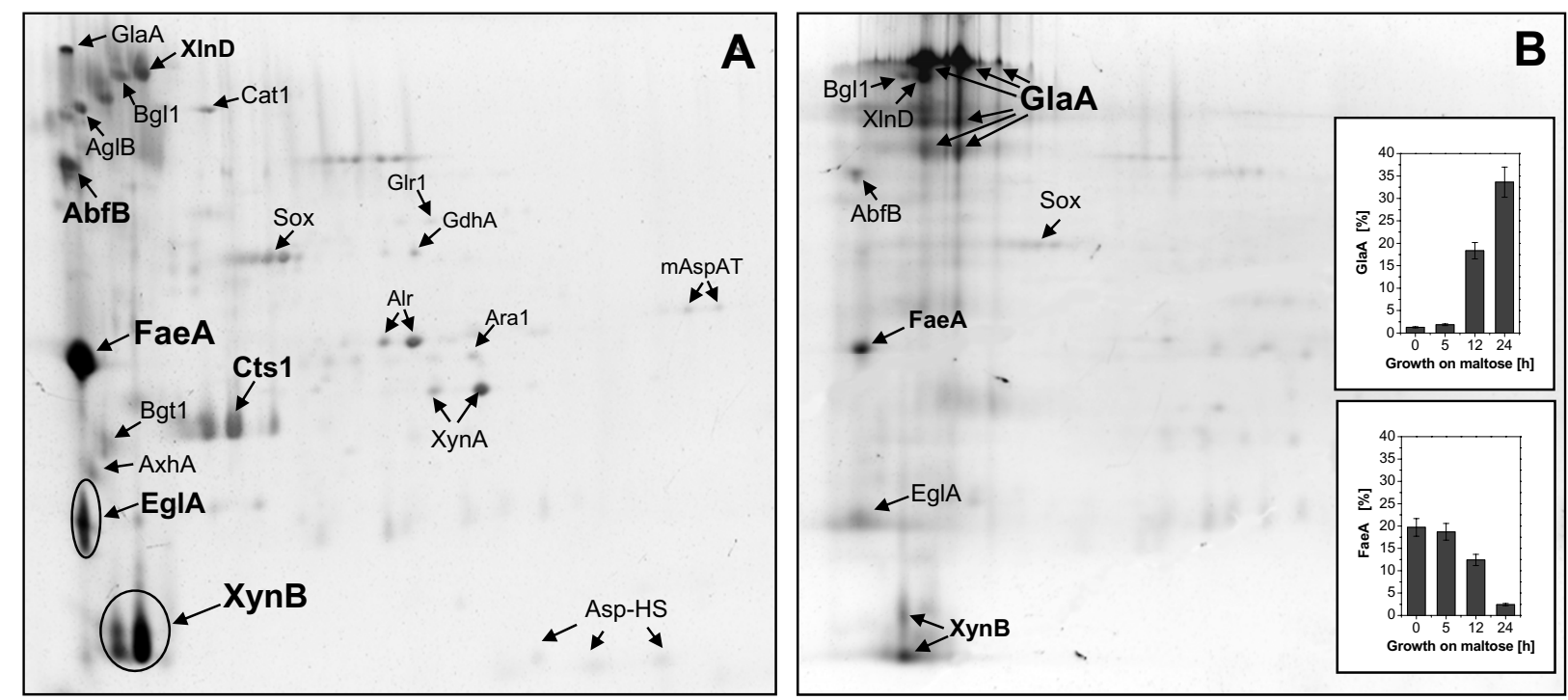

Figure 4 Change of extracellular proteome in response to maltose addition to a culture pregrown on xylose. Change of the extracellular proteome of A. niger AB1.13 in response to maltose addition to a bioreactor culture pregrown on xylose. (A) The composition of the extracellular proteome of cells grown on defined medium with xylose as carbon substrate. (B) Change of the extracellular proteome $24 \mathrm{~h}$ after the addition of maltose to a culture pregrown on xylose. Characteristics of proteins indicated by arrows are discussed in more detail. The basic side of the gel is on the right. The inserts on the right show the time-dependent fraction change of two selected proteins from the extracellular proteome after the addition of maltose. A detailed list of all proteins from the extracellular proteome of A. niger growing either on xylose or maltose identified on 2-D gels is found in Additional file 6 
depending on the carbon substrate is found in Additional file 5 .

\section{Intracellular proteins found in higher abundance in the maltose-grown culture}

The only identified protein found exclusively in the maltose grown culture was glucose oxidase (GoxC) (Fig. 3, Additional file 5). This enzyme catalyzes the oxidative conversion of glucose, the product of maltose hydrolysis, into gluconic acid and hydrogen peroxide. In this line, enzymes involved in the removal of reactive oxygen species, such as superoxide dismutase (SodC) or peroxiredoxin (PrxII) were found in much higher abundance in the culture growing on maltose. An unexpected protein more abundant in the mycelium of the maltose grown culture was the protein folding catalyst cyclophilin-like peptidyl prolyl cis-trans isomerase (CypA). For a detailed list of all identified intracellular proteins showing significant changes in abundance depending on the carbon substrate see Additional file 5.

\section{The extracellular proteome of A. niger grown on xylose or maltose in bioreactor cultures}

The composition of the intracellular proteome of A. niger growing either on maltose or xylose in controlled bioreactor cultures revealed striking similarities. The extracellular proteome, however, showed considerable differences depending on the utilisation of xylose or maltose as carbon substrate (Fig. 4 and Additional files 6 and 7).

A total of 21 proteins were identified in the extracellular proteome of $A$. niger growing on xylose by $2-\mathrm{D}$ gel electrophoresis in combination with Maldi-ToF analysis (Fig. 4a, Additional file 6). The majority of these extracellular proteins are hydrolytic enzymes involved in the degradation of cell wall polymers, such as xylanolytic enzymes, namely the endo-1,4- $\beta$-xylanases $A$ and $B$ (XynA and XynB, respectively) and $\beta$-xylosidase $(X \ln D)$, arabinoxylan degrading enzymes such as 1,4- $\beta$-D-arabinoxylan arabinofuranohydrolase (AxhA) and arabinofuranosidase $B(A b f B)$, as well as cellulolytic enzymes such as endoglucanase (EglA) and $\beta$-glucosidase (Bgl1). Moreover, the lactose hydrolysing $\alpha$-galactosidase (AglB) and also small quantities of the starch-degrading glucoamylase (GlaA) were found in the culture medium. XynB (appearing in three different spots) and ferulic acid esterase (FaeA), an enzyme which can remove aromatic compounds (e.g. ferulic acid) from plant cell wall polysaccharides (pectin and xylan), represented the two most abundant extracellular proteins accounting for more than $30 \%$ of the entire extracellular proteome. Altogether, all extracellular hydrolases represent about $60 \%$ of the extracellular proteome of $A$. niger during growth on xylose. The majority of genes encoding these extracellular hydro- lases $(x y n A, x y n B, x \ln D, a x h A, f a e A$, eglA) are controlled by the xylanolytic transcriptional activator $X \ln R[26,28]$.

Other proteins found in the medium of $A$. niger grown on xylose are 3- $\beta$-glucanosyltransferase (Bgt1) and chitinase (Cts1). Bgt1 is responsible for the elongation of 1,3$\beta$-glucan chains during cell wall synthesis [35] and chitinases are produced by filamentous fungi which have chitin as a cell wall component and are considered to play a pivotal role during autolysis of the fungal cell wall [3638]. Moreover, enzymes generating or removing reactive oxygen species such as sulfhydryl oxidase (Sox), which catalyses the conversion of reduced glutathione to its disulfide form with concomitant consumption of oxygen and release of hydrogen peroxide [39], or catalase (Cat1) which protects cells against oxidative damage caused by hydrogen peroxide through its catalytic decomposition [40], were identified in the extracellular proteome of $A$. niger during growth on xylose. Moreover, a putative Asphemolysin (Asp-HS), a protein who's homolog in $A$. fumigatus is a virulence factor and cytolytic toxin [41], was found in the extracellular proteome during growth on xylose. Some other clearly intracellular proteins such as an $\mathrm{NADPH}$ dependent glutamate dehydrogenase (GdhA), glutathione reductase (Glr1), D-arabinose dehydrogenase (Ara1), the NADPH dependent aldehyde reductase (Alr) and the mitochondrial aspartate aminotransferase (mAspAT) were also detected as minor components of the extracellular proteome most likely as a result of cell lysis.

The extracellular proteome of $A$. niger grown on maltose (Additional file 7) is dominated by glucoamylase, which accounts for more than $50 \%$ of the extracellular proteome and appears in multiple spots. Components of the extracellular proteome of the maltose-grown culture which were also identified in the culture grown on xylose include, for example, $\beta$-glucosidase (Bgl1), sulfhydryl oxidase (Sox) and catalase (Cat1). Cat1 was found in much higher abundance in the extracellular proteome of $A$. niger growing on maltose. Moreover, another catalase (CatR) was additionally identified in the extracellular proteome of $A$. niger growing on maltose but not in the xylose grown culture, additionally reflecting the increased demand for removal of reactive oxygen species such as hydrogen peroxide in the maltose-grown culture. Few proteases, including small amounts of PepA, were identified in the extracellular proteome, but only in the culture grown on maltose, consistent with the fact that $A$. niger $\mathrm{AB} 1.13$ is a mutant protease-reduced strain which has only $1-2 \%$ of the extracellular proteolytic activity of the parental strain $[42,43]$. Extracellular hydrolases controlled by the xylanolytic transcriptional activator $X \ln R$ (xynA, xynB, xlnD, axhA, faeA, eglA) were completely absent in the extracellular proteome of $A$. niger $\mathrm{AB} 1.13$ 
when cells were grown on maltose (Additional file 7). A detailed list of all identified proteins from the extracellular proteome of $A$. niger growing either on xylose or maltose identified on 2-D gels or identified by nano-HPLC MS/MS (in total 69 proteins) is found in Additional file 6.

To study the change in the composition of the extracellular proteome after induction of glaA-promoter controlled protein production maltose was added to a culture pregrown on xylose and the change in the composition analyzed by 2-D electrophoresis (Fig. 4). The proteome changed drastically after the addition of maltose, the fraction of xylanolytic enzymes declined strongly and glucoamylase (appearing in multiple spots) began to dominate reaching a fraction of more than $30 \%$ in the secretome $24 \mathrm{~h}$ after the addition of maltose (Fig. 4B). Catalases did not accumulate to detectable levels in the extracellular proteome in response to the addition of maltose to the culture pregrown on xylose. This is in contrast to the increased level of catalases found in the extracellular proteome of the culture which was growing on maltose from the beginning (Additional file 7). In this line high levels of glucose and gluconate were formed in the latter while late addition of maltose to the culture pregrown on xylose did not lead to detectable formation of glucose and gluconate (data not shown).

\section{Comparative analysis of the intracellular proteome of A. niger grown on xylose in controlled bioreactor or shake flask culture}

Most often $A$. niger is grown in shake flask culture without the possibility to control $\mathrm{pH}$ and provide sufficient oxygen through controlled airation and agitation. This difference in growth conditions can translate into different productivites most often with considerable lower product concentrations obtainable in shake flask cultures. Using the same inoculum and the same medium with xylose as carbon substrate, similar general growth kinetics were observed in bioreactor and shake flask cultures (Fig. 1). The final biomass, however, was considerably lower in shake flask culture reaching only appr. $80 \%$ of the biomass obtainable in controlled bioreactor culture.

The intracellular proteome of $A$. niger growing to late exponential/early stationary phase on the same xylose containing defined medium either in controlled batch bioreactor or shake flask culture was surprisingly different (Fig. 5, Additional file 8). Abundant proteins present in the mycelium of the bioreactor culture such as the glycolytic enzymes Fba1 and GpdA and the TCA cycle enzyme Mdh1 were only present in minor amounts in the mycelium of the shake flask culture indicating a reduced capacity of the glycolytic pathway and the TCA cycle under these conditions. Also, the flavohemoglobin FhbA and the enzymes involved in the biosynthesis of thiamine,
Nmt1 and Nmt2, all prominent proteins in the bioreactor culture with putative roles in the oxidative stress response and oxidative damage tolerance, were not or only in minor amounts present in the shake flask culture probably indicating the reduced oxygen supply in shaker flasks. In shake flask culture, however, the NADPHdependent aldehyde reductase (Alr) represented one of the major components of the intracellular proteome. Also, SodC was one of the most abundant proteins in the shake flask grown culture but only a faint spot on the 2-D gel from the bioreactor culture sample. In this line, a catalase (CpeB) was only found in the intracellular proteome of the shake flask but was not present in the intracellular proteome of the bioreactor culture indicating that cells experience more oxidative stress from reactive oxygen species (radicals and peroxides) during shake flask cultivation in contradiction to the reduced oxygen availability in shaker flask cultures. Moreover, fungal proteases such as the vacuolar proteases $\mathrm{PepC}$, a subtilisin-type serine endoprotease [44], and PepE, an aspartic pepsin-like endoprotease [45], Hex1 (a structural component of the fungal Woronin body, [46]), or an $\mathrm{NAD}^{+}$-dependent formate dehydrogenase of yet unclear physiological function (Fdh, formerly known as AciA [47-49]) were found in higher quantities in the shake flask culture (Fig. 5, Additional file 8 ). The most abundant protein, however, present in the mycelium of $A$. niger growing in the shake flask culture but only present as a minor protein in the mycelium of the bioreactor culture was identified as An07g03660 (similar to An09g00630) a CipC-like protein of yet unknown function (Fig. 5, Additional file 8). The name CipC derives from concanamycin-induced protein because its orthologue in $A$. nidulans is upregulated in response to the antibiotic concanamycin A [50]. Moreover, in A. fumigatus CipC was identified as one of the major conodial surface associated proteins [51].

In general, glycolytic and TCA cycle enzymes and many chaperones and foldases (e.g. BipA, Ssb2, Hsp60, TpiA) accumulated to significantly higher levels in the bioreactor culture, while in shake flask cultures vacuolar proteases (PepE and PepC) and (oxidative) stress proteins involved in the removal of reactive oxygen species and other proteins of more diverse function were of higher abundance. Thus, the difference in culture conditions ( $\mathrm{pH}$ control versus no $\mathrm{pH}$ control, aeration versus no aeration and stirring versus shaking) has a more profound effect on the metabolic status of the cells and the composition of the intracellular proteome than the carbon substrate. For a detailed list of all identified intracellular proteins showing significant changes in abundance during growth in bioreactor or shake flask culture see Additional file 8 . 


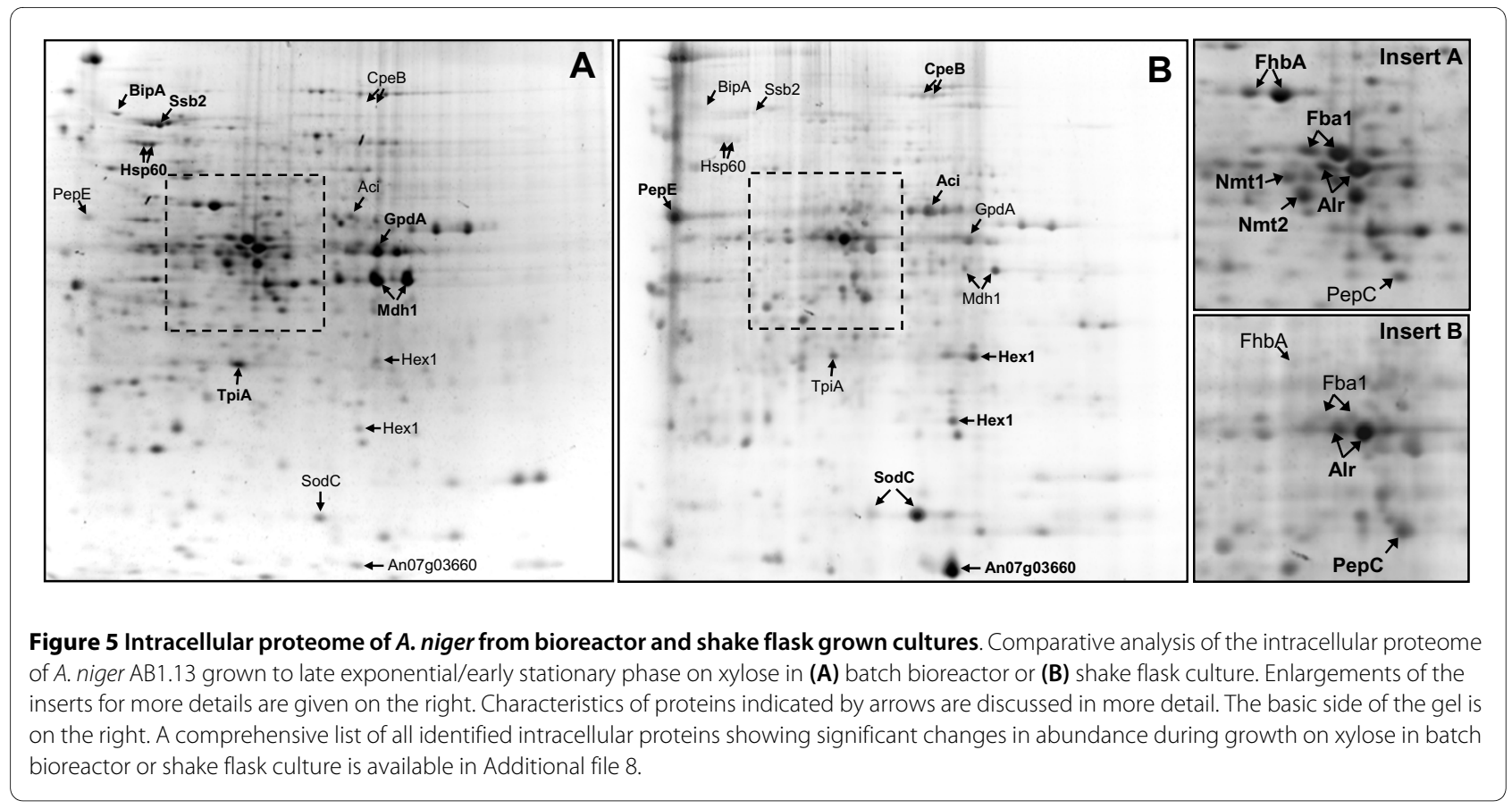

\section{Conclusions}

\section{Intracellular proteome}

The comparative analysis of the intracellular proteome of A. niger grown either on xylose or maltose in controlled batch bioreactor cultures revealed striking similarities. The glycolytic enzymes Fba1 and GpdA and the TCA cycle enzyme Mdh1 were among the most abundant proteins and accumulated to comparable amounts in both cultures. Past enzyme activity measurements in continuous cultures of $A$. niger revealed malate dehydrogenase as the most active enzyme of the TCA cycle with an activity steadily increasing with increasing dilution rate [52]. Thus, similar and elevated amounts of Mdh1 during batch growth on xylose and maltose in bioreactor culture might reflect the comparable high growth rates obtained on these substrates and indicate that energy metabolism and not the initial steps in xylose or maltose breakdown are controlling the final growth rate. In this line, a transcriptomic comparison of $A$. niger growing either on xylose or maltose in carbon-limited continuous cultures at identical dilution rate also revealed little differences in the expression of genes from central metabolic processes [53].

Moreover, comparable high amounts of GpdA in both xylose and maltose grown batch bioreactor cultures also explain previous observations that $g p d A$ promoter controlled production of recombinant proteins leads to similar amounts of the recombinant product in controlled bioreactor cultures using either glucose or xylose as carbon substrate [54].

ER-resident chaperones and foldases were present in both cultures in similar amounts indicating a comparable capacity for protein secretion under both conditions. The transcriptomic analysis of $A$. niger growing either on xylose or maltose in carbon-limited continuous cultures indicated a modest but significant higher expression of secretory pathway genes during growth on maltose [53]. This discrepancy to our observation of equal abundance of ER-resident chaperones and foldases in both xylose and maltose batch cultures is difficult to evaluate as it might be due to the different strains and culture conditions employed. Moreover, a modest increase in transcript level also does not necessarily translate into a detectable increase in protein amount. Yet, these results clearly show the need for a combined transcriptome and proteome analysis.

Strong differences in the intracellular proteome of $A$. niger growing on xylose or maltose are related to the first steps in carbon substrate catabolism. The culture growing on xylose exclusively contained D-xylose reductase and some plant cell-wall (e.g. xylan, arabinoxylan) degrading enzymes while glucose oxidase was only found in the culture using maltose as carbon substrate. Most strikingly were the differences in the amount of intracellular fungal proteins involved in the oxidative stress response; growth on maltose was clearly leading to higher levels of enzymes involved in the removal of reactive oxygen species indicating higher oxidative stress during maltose/glucose breakdown.

\section{Extracellular proteome}

The composition of the extracellular proteome was completely different for both bioreactor cultures. A. niger growing on xylose secreted mainly hydrolases involved in the degradation of plant cell wall polymers while the 
extracellular proteome of $A$. niger growing on maltose was dominated by glucoamylase. Again, enzymes involved in the removal of reactive oxygen species were more abundant in the extracellular proteome during growth on maltose. In this culture elevated glucose concentrations were observed leading to glucose oxidase (GoxC) synthesis and subsequent oxidation of glucose to gluconic acid and hydrogen peroxide, thus explaining the need for catalases to remove these reactive oxygen species. Late addition of maltose to the culture pregrown on xylose did not lead to elevated levels of extracellular enzymes involved in the removal of reactive oxygen species (e.g. catalases) as the formed glucose resulting from the hydrolysis of maltose will be taken up by the existing fungal biomass and not further oxidized to gluconic acid and hydrogen peroxide, thus making elevated catalase levels dispensable.

\section{Bioreactor versus shake flask}

The major differences influencing fungal physiology in bioreactor versus shake flask culture are related to $\mathrm{pH}$ control versus no $\mathrm{pH}$ control, good aeration versus bad aeration, and higher mechanical stress due to stirring versus lower mechanical stress due to shaking. This difference in the environmental conditions had a profound effect on the composition of the intracellular proteome. In uncontrolled shake flask cultures, the major glycolytic and TCA cycle enzymes were only present in minor amounts indicating a reduced capacity of the glycolytic pathway and the TCA cycle under these conditions. Moreover, ER-resident chaperones and foldases were present in lower amounts while vacuolar proteases accumulated to higher levels in shake flask culture rendering this culture condition less favourable for recombinant protein production. In this line, previous results also revealed that higher levels of recombinant protein product are generally obtained in bioreactor cultures compared to uncontrolled shake flask cultivations $[55,56]$.

Our results also showed that many proteins found in high abundance under certain culture conditions are still proteins with yet unclear physiological function. Though we are still far from understanding their role, this work now offers more tools for a better understanding of fungal physiology and, thus, for more rational based approaches to improve the productivity of this black fungus.

\section{Materials and methods \\ Strain and medium}

A. niger AB1.13, an uridine auxotroph, glucoamylase producing strain, was first described as deficient in aspergillopepsin A (PepA) and aspergillopepsin B (PepB) with residual proteolytic activity attributable to the presence of low levels of PepA or the activity of another pepstatinsensitive protease [42]. A. niger AB1.13 was generated by
UV mutagenesis of $A$. niger AB4.1 and subsequent selection for reduced extracellular protease activity [42]. $A$. niger AB4.1 is a pyrG mutant (requiring uridine or uracil for growth) of $A$. niger $\mathrm{N} 402$ [57], which is a $\operatorname{csp} A$-derivative (short conidiophores) of $A$. niger ATCC9029 (= NRRL3) [58]. More recently, it was shown that $A$. niger $\mathrm{AB} 1.13$ is a regulatory mutant which carries a mutation in the $\operatorname{prt} T$ gene, a protease specific regulator for the fungal $\mathrm{Zn}$ (II)2Cys6 cluster transcription factor family, being responsible for its reduced extracellular proteolytic activity [43].

A. niger AB1.13 was grown on a synthetic medium containing either xylose or maltose as carbon source. The defined medium was a modified Vogel' medium [59] supplemented with $2.44 \mathrm{~g} \mathrm{~L}^{-1}$ uridine. The composition was as follows: $\mathrm{Na}_{3}$ citrate $\cdot 2 \mathrm{H}_{2} \mathrm{O}, 2.85 \mathrm{~g} \cdot \mathrm{L}^{-1} ; \mathrm{KH}_{2} \mathrm{PO}_{4}, 5 \mathrm{~g} \cdot \mathrm{L}^{-1}$; $\mathrm{NH}_{4} \mathrm{NO}_{3}, 2 \mathrm{~g} \cdot \mathrm{L}^{-1} ; \mathrm{CaCl}_{2} \cdot 2 \mathrm{H}_{2} \mathrm{O}, 0.1 \mathrm{~g} \cdot \mathrm{L}^{-1} ; \mathrm{MgSO}_{4} \cdot 7 \mathrm{H}_{2} \mathrm{O}, 0.2$ $\mathrm{g} \cdot \mathrm{L}^{-1}$ and trace elements (citric acid $\cdot \mathrm{H}_{2} \mathrm{O}, 5 \mathrm{mg} \cdot \mathrm{L}^{-1}$; $\mathrm{ZnSO}_{4} \cdot 7 \mathrm{H}_{2} \mathrm{O}, 5 \mathrm{mg} \cdot \mathrm{L}^{-1}$; $\mathrm{Fe}\left(\mathrm{NH}_{4}\right)_{2}\left(\mathrm{SO}_{4}\right)_{2} \cdot 6 \mathrm{H} 2 \mathrm{O}, 1 \mathrm{mg} \cdot \mathrm{L}^{-1}$; $\mathrm{CuSO}_{4}, 0.16 \mathrm{mg} \cdot \mathrm{L}^{-1} ; \mathrm{MnSO} 4 \cdot \mathrm{H}_{2} \mathrm{O}, 0.037 \mathrm{mg} \cdot \mathrm{L}^{-1} ; \mathrm{H}_{3} \mathrm{BO}_{3}$, $\left.0.05 \mathrm{mg} \cdot \mathrm{L}^{-1} ; \mathrm{Na}_{2} \mathrm{MoO}_{4}, 2 \mathrm{H}_{2} \mathrm{O}, 0.05 \mathrm{mg} \cdot \mathrm{L}^{-1}\right)$. The initial concentration of carbon source, either xylose or maltose, was $20 \mathrm{~g} \cdot \mathrm{L}^{-1}$. For the study of the change in the composition of the extracellular proteome after induction of glaApromoter by addition of maltose to a culture pregrown on xylose, cultivation was started with an initial concentration $10 \mathrm{~g} \cdot \mathrm{L}^{-1}$ xylose and after $68 \mathrm{~h}$ of growth on xylose maltose was added to a final concentration of $10 \mathrm{~g} \cdot \mathrm{L}^{-1}$.

\section{Inoculation, culture conditions, and analytical procedures}

Inoculation was carried out by $1 \times 10^{7}$ spores $\mathrm{mL}^{-1}$ obtained from a densely conidiating culture grown on $3.8 \%(\mathrm{w} / \mathrm{v})$ potato dextrose agar (PDA) supplemented with $2.44 \mathrm{~g} \mathrm{~L}^{-1}$ uridine at $30^{\circ} \mathrm{C}$ and harvested after 5 days with sterile $\mathrm{NaCl}$ solution $(0.9 \% \mathrm{w} / \mathrm{v})$. The spore numbers in the suspension were counted using a hemocytometer.

Shake flask cultivations were carried out in $500 \mathrm{~mL}$ Erlenmeyer flasks with 4 baffles on a rotary shaker (Pilot Shaker Rc 6SR, B. Braun Diesel Biotech GmbH, Melsungen, Germany) at $30^{\circ} \mathrm{C}$ and $120 \mathrm{rpm}$, and $100 \mathrm{~mL}$ working volume. Initial $\mathrm{pH}$ was adjusted to 5.5 .

Batch cultivations were carried out in a $12 \mathrm{~L}$ stirred tank bioreactor Biostat ${ }^{\circ} \mathrm{C}$ (B.Braun international) with a working volume of $10 \mathrm{~L}$ at $30^{\circ} \mathrm{C}$. The stirrer of the Biostat ${ }^{\circ} \mathrm{C}$ was equipped with three 6 -bladded rushton turbine impellers. The stirrer speed and the aeration rate were $300 \mathrm{rpm}$ and $1 \mathrm{vvm}$, respectively. The $\mathrm{pH}$ was controlled at pH 5.5 using $20 \%$ (w/v) $\mathrm{NaOH}$. Foam was suppressed by the manual addition of antifoam reagent Ucolub (Th. Goldschmidt AG, Essen, Germany).

Cell dry mass (CDM) was determined from 10-mL aliquots of culture broth. The fungal biomass was collected by filtration through a filter paper (Sartorius, grade: 389), 
washed twice with distilled water and dried overnight at $40^{\circ} \mathrm{C}$ under vacuum to constant weight.

\section{Preparation of the intracellular protein fraction}

Biomass (around $500 \mathrm{mg}$ wet biomass) was harvested by filtration (filter paper: Sartorius, grade: 389 ) and washed with phosphate buffered saline (PBS) to remove extracellular proteins and other contaminants. Cell disruption was accomplished by grinding the wet biomass in liquid nitrogen using a Mortar Grinder (RM 100, Retsch GmbH \&Co. KG, Germany). After cell disruption, the biomass was resuspended in $1 \mathrm{ml}$ extraction buffer $\left(20 \mathrm{mmol} \mathrm{L}^{-1}\right.$ Tris- $\mathrm{HCl}$, pH 7.6, $10 \mathrm{mmol} \mathrm{L}^{-1} \mathrm{NaCl}, 0.5 \mathrm{mmol} \mathrm{L}^{-1}$ deoxycholate, $1 \mu \mathrm{g} \mathrm{mL}^{-1}$ pepstatin). Cell debris was removed by centrifugation $\left(22,000 \times \mathrm{g}\right.$, at $4^{\circ} \mathrm{C}$ for $\left.30 \mathrm{~min}\right)$ and the subsequent supernatant treated for $15 \mathrm{~min}$ on ice with $7 \mu \mathrm{L}$ nuclease mix $\left(0.5 \mathrm{mg} \mathrm{mL}^{-1}\right.$ DNase, $0.25 \mathrm{mg} \mathrm{mL}^{-1}$ RNase, und $\left.50 \mathrm{mmol} \mathrm{L}^{-1} \mathrm{MgCl}_{2}\right)$. Then, $10 \mu \mathrm{L} \mathrm{mL}-1$ sodium deoxycholate $\left(20 \mathrm{mg} \mathrm{mL}^{-1}\right)$ were added and the mixture kept for at least $30 \mathrm{~min}$ on ice followed by addition of TCA to a final concentration of $12 \%(\mathrm{w} / \mathrm{v})$. This mixture was incubated for at least $1 \mathrm{~h}$ at $4^{\circ} \mathrm{C}$ and the precipitated proteins were collected by centrifugation at $22,000 \times \mathrm{g}$ for $1 \mathrm{~h}$ at $4^{\circ} \mathrm{C}$. The protein pellet was washed with $300 \mu \mathrm{L}$ ice cold acetone to remove traces of TCA, centrifuged, washed again with acetone, and solubilized in solubilization buffer containing $7 \mathrm{~mol} \mathrm{~L}^{-1}$ urea, $2 \mathrm{~mol} \mathrm{~L}^{-1}$ thiourea, 4\% (w/v) CHAPS, 1\% (w/v) DTT, $20 \mathrm{mmol} \mathrm{L}^{-1}$ Tris, and $1 \%$ (v/v) Pharmalyte ${ }^{\mathrm{mm}} \mathrm{pH}$ 3-10 (Amersham Biosciences). The total soluble protein concentration was determined according to the method of Bradford [60] using the BIORAD protein assay (BIO-RAD Lab., Hartfordshire, USA). The solubilized proteins were stored at $-70^{\circ} \mathrm{C}$ until further analysis by two-dimensional gel electrophoresis.

\section{Preparation of the extracellular protein fraction}

For preparation of the extracellular protein fraction, biomass was removed by centrifugation at $6,000 \times \mathrm{g}$ for 15 $\min$ at $4^{\circ} \mathrm{C}$ from the culture broth and the supernatant filtered through a $0.2 \mu \mathrm{m}$ filter. Protein precipitation, solubilization, and storage were carried out as described above.

\section{Two-dimensional gel electrophoresis}

The first-dimension using isoelectric focusing (IEF) was run with the IPGphor ${ }^{\text {ma }}$ Isoelectric Focusing System (Amersham Biosciences) at $20^{\circ} \mathrm{C}$ with a current of $45 \mu \mathrm{A}$ per strip. $300 \mu \mathrm{g}$ of each protein sample were loaded onto Immobiline DryStrip gels of pH 3-10 (IPG strips, Amersham Biosciences) by in-gel rehydration. IEF was performed with the following conditions: $30 \mathrm{~V} \times 12 \mathrm{~h}, 300 \mathrm{~V}$ $\times 3 \mathrm{~h}, 600 \mathrm{~V} \times 2 \mathrm{~h}, 1000 \mathrm{~V} \times 1 \mathrm{~h}$, gradient from $1000 \mathrm{~V}$ to $5000 \mathrm{~V}$ within $2 \mathrm{~h}, 5000 \mathrm{~V} \times 2 \mathrm{~h}$, gradient from $5000 \mathrm{~V}$ to $8000 \mathrm{~V}$ within $2 \mathrm{~h}$, then $8000 \mathrm{~V} \times 10 \mathrm{~h}$. Prior to the second dimension (SDS-PAGE), the IPG strips were equilibrated for $15 \mathrm{~min}$ in $6 \mathrm{~mol} \mathrm{~L}^{-1}$ urea, 30\% glycerol (v/v), 2\% SDS (w/v), $50 \mathrm{mmol} \mathrm{L}^{-1}$ Tris-HCl pH 8.8, containing 1\% (w/v) DTT and then for $15 \mathrm{~min}$ in the same buffer additionally containing $2.5 \%(\mathrm{w} / \mathrm{v})$ iodoacetamide. Equilibrated strips were transferred for the second dimension onto lab cast SDS-polyacrylamide gels (DALT multiple gel caster and DALT gradient maker, Amersham Biosciences). Intraand extracellular proteins were separated on $12-16 \%$ linear gradient and $12 \%$ uniform gels, respectively. The second-dimension was carried out using the vertical separation unit Hoefer ${ }^{\text {rm }}$ System (Amersham Biosciences) at $10^{\circ} \mathrm{C}$ in constant working voltage mode as follows: $80 \mathrm{~V}$ for $1 \mathrm{~h}$ and then $125 \mathrm{~V}$ overnight until the bromophenol blue dye front reached the bottom of the gel. Subsequently, gels were stained using colloidal Coomassie Blue G-250 according to the "Blue silver" protocol [61]. The gels were then scanned (ScanMaker 9800 XL, Umax System $\mathrm{GmbH}$, Germany) at $300 \mathrm{dpi}$ resolution to acquire the gel images. Image analysis, namely protein spot detection, matching and quantification were performed using Proteomweaver ${ }^{\mathrm{Tm}}$ 3.0 (Definiens AG, Germany).

\section{Identification of protein spots from two-dimensional gels}

Protein spots were excised manually from the stained gels. After destaining, reduction and alkylation, in-gel digestion was carried out by incubation with $2 \mathrm{ng} \mu \mathrm{L}^{-1}$ trypsin (sequencing grade modified, Promega Corp.) in $50 \mathrm{mmol} \mathrm{L}-1$ ammonium bicarbonate at $37^{\circ} \mathrm{C}$ overnight. Obtained peptides were extracted and then purified with reversed-phased C18 ZipTips (Millipore, USA) as described previously [62]. MALDI-ToF and MALDI ToF/ ToF for mass mapping of tryptic peptides were carried out using a Bruker Ultraflex time-of-flight mass spectrometer (Bruker Daltonics GmbH, Germany). Nanoelectrospray ionisation quadrupole-time-of-flight tandem mass spectrometry (ESI-QqTOF MS/MS) was performed using a Q-TOF2 mass spectrometer (Micromass, Manchester, England) to obtain partial amino acid sequences. Peptide mass fingerprints and peptide fragmentation data were processed using FlexAnalysis 2.0 (Bruker Daltonik $\mathrm{GmbH}$, Germany) and the PepSeq program of the software package Masslynx ${ }^{\mathrm{Tw}}$ (Waters Corporation, USA). The MASCOT 2.1.0 search program (Matrix Science, UK) was used for protein identification with the annotated Aspergillus niger genome ([11], EMBL: http:// www.ebi.ac.uk/genomes/eukaryota.html) serving as database. The search parameters were set as follows: tryptic digestion, one missed cleavage side allowed, carbamidomethylation of cysteine (fixed modification), methionine oxidation (variable modification), all peptides monoisotopic, peptide tolerance at $20 \mathrm{ppm}$. All proteins with a Mowse score greater than 54 were regarded as significant $(\mathrm{p}<0.05)$. Annotations are according to the 
sequenced genome of $A$. niger [11] and the current NCBI Reference Sequence database (http:// www.ncbi.nlm.nih.gov/refseq/, status 2010/03/26).

\section{Nano-HPLC MS/MS}

HPLC MS/MS was additionally applied for the analysis of the extracellular proteome of Aspergillus niger AB1.13. In this case, proteins were first separated by $12 \%$ SDS-PAGE (10 mm distance). After staining with Coomassie brilliant blue, the gel was cut into slices. Protein digestion and peptide isolation was carried out as described for MALDI samples. The separation of the peptide samples was performed using a bioinert Ultimate nano-HPLC system (Dionex, Sunnyvale, CA) as previously described [63]. MS and MS/MS data were acquired using a Q-TOF II mass spectrometer (Waters Corp., Micromass, Manchester, UK). Doubly and triply charged peptide-ions were automatically chosen by the MassLynx software and fragmented for a maximum of $18 \mathrm{~s}$ for each peptide. MS data processing and generation of peptide peak lists were also carried out using MassLynx software (Waters Corporation, Manchester, UK). Identification was regarded as valid with a significance value of $\mathrm{p}<0.05$.

\section{Additional material}

Additional file 1 Biochemical reactions with corresponding enzymes. Biochemical reactions and corresponding enzymes (coding sequences CDSs) potentially catalyzing these reactions. Reactions and CDSs depicted in Figure 2 are indicated. Moreover, all CDSs experimentally verified by 2-D GE and MALDI ToF or LC-MS/MS are indicated.

Additional file $\mathbf{2}$ All identified intracellular proteins. Classification of intracellular proteins of $A$. niger AB1.13 grown on defined medium with xylose or maltose as carbon substrate that were identified via 2-D GE followed by MALDI ToF.

Additional file 3 Interactive 2-D gel of intracellular proteome of $\boldsymbol{A}$. niger grown on xylose. Image of 2-D gel for detailed analysis of the intracellular proteome of $A$. niger AB1.13 grown to late exponential/early stationary phase in bioreactor cultures on defined medium with xylose as carbon substrate. The red spots indicate proteins identified by Maldi-ToF analysis. More information on these proteins can be found by moving the mouse over the spots (for locci ID, function, MW and pl, tested with Windows Internet Explorer), or by clicking them to visit the NCBI database http:// www.ncbi.nlm.nih.gov/ for their detailed annotation. Please note that the compressed zip file has to be unpacked locally (double click is not sufficient) after downloading.

Additional file 4 Interactive 2-D gel of intracellular proteome of $A$. niger grown on maltose. Image of 2-D gel for detailed analysis of the intracellular proteome of A. niger AB1.13 grown to late exponential/early stationary phase in bioreactor cultures on defined medium with maltose as carbon substrate. The red spots indicate proteins identified by Maldi-ToF analysis. More information on these proteins can be found by moving the mouse over the spots (for locci ID, function, MW and pl, tested with Windows Internet Explorer), or by clicking them to visit the NCBI database http://www.ncbi.nlm.nih.gov/ for their detailed annotation. Please note that the compressed zip file has to be unpacked locally (double click is not sufficient) after downloading.

Additional file $\mathbf{5}$ Abundance difference of intracellular proteins from xylose and maltose grown cultures. Identified intracellular proteins showing significant changes in abundance during growth in bioreactor culture using xylose or maltose as carbon substrate.
Additional file 6 All identified extracellular proteins. Classification of extracellular proteins of $A$. niger AB1.13 grown on defined medium with xylose or maltose as carbon substrate that were identified via 2-D GE followed by MALDI ToF or LC-MS/MS.

Additional file 7 Extracellular proteome of $A$. niger grown on xylose or maltose. Comparative analysis of the extracellular proteome of $A$. niger AB1.13 grown in bioreactor cultures on defined medium with (A) xylose or (B) maltose as carbon substrate. Characteristics of proteins indicated by arrows are discussed in more detail in the main body text. The basic side of the gel is on the right. A detailed list of all proteins from the extracellular proteome of A. niger growing either on xylose or maltose identified on 2-D gels is found in Additional file 6 .

Additional file 8 Abundance difference of intracellular proteins from bioreactor and shake flask grown cultures. Identified intracellular proteins showing significant changes in abundance during growth on xylose in bioreactor or shake flask culture.

\section{Competing interests}

The authors declare that they have no competing interests.

\section{Authors' contributions}

$\mathrm{XL}$ carried out all experimental work and prepared a first draft of the manuscript. JS did the metabolic network analysis and prepared the interactive figures in HTML format, MN and JW contributed to protein identification by Maldi-ToF and Nano-HPLC MS/MS, respectively. APZ was involved in the initial work on network analysis, UR initiated and supervised the study and wrote the final manuscript. All authors read and approved the final manuscript.

\section{Acknowledgements}

This study was carried out in part in the framework of the Sonderforschungsbereich 578 (Project B1/B4). We gratefully acknowledge access to the DSM A. niger database prior to public release, in particular the excellent support provided by Hans Roubos, Rob van der Hoeven, Noel van Peij and Gert Groot. We also gratefully acknowledge the comments of the reviewers which helped to improve the manuscript. Moreover, we are also grateful to Peter Punt who pointed our attention to recent publications on the flavohemoglobin.

\section{Author Details}

Helmholtz Center for Infection Research, Inhoffenstr, 7, 38124 Braunschweig, Germany

Received: 22 January 2010 Accepted: 20 April 2010

Published: 20 April 2010

\section{References}

1. Finkelstein DB: Improvement of enzyme production in Aspergillus. Antonie van Leeuwenhoek 1987, 53:349-352.

2. Nunberg JH, Meade JH, Cole G, Lawyer FC, McCabe P, Schweickart V, Tal R, Wittman VP, Flatgaard JE, Innis MA: Molecular cloning and characterization of the glucoamylase gene of Aspergillus awamori. Mol Cell Biol 1984, 4:2306-2315.

3. Fowler T, Berka RM, Ward M: Regulation of the glaA gene of Aspergillus niger. Curr Genet 1990, 18:537-545.

4. Carrez D, Janssens W, Degrave P, Hondel CAMJJ van den, Kinghorn JR, Fiers W, Contreras R: Heterologous gene expression by filamentous fungi: secretion of human interleukin- 6 by Aspergillus nidulans. Gene 1990, 94:147-154

5. Hondel CAMJJ van den, Punt PJ, van Gorcom RFM: Production of extracellular proteins by the filamentous fungus Aspergillus. Antonie van Leeuwenhoek 1991, 61:153-160.

6. Schrickx JM, Krave AS, Verdoes JC, Hondel CAMJJ van den, Stouthamer $\mathrm{AH}$, van Verseveld HW: Growth and product formation in chemostat and recycling cultures by Aspergillus niger N402 and a glucoamylase overproducing transformant, provided with multiple copies of the glaA gene. J Gen Microbiol 1993, 139:2801-2810.

7. Verdoes JC, Punt PJ, Stouthamer AH, Hondel CAMJJ van den: The effect of multiple copies of the upstream region on expression of the Aspergillus niger glucoamylase-encoding gene. Gene 1994, 145:179-187.

8. Siedenberg D, Mestric S, Ganzlin M, Schmidt M, Punt PJ, Hondel CAMJJ van den, Rinas U: GlaA promoter controlled production of a mutant 
green fluorescent protein (S65T) by recombinant Aspergillus niger during growth on defined medium in batch and fed-batch cultures. Biotechnol Prog 1999, 15:43-50

9. Ganzlin M, Rinas U: In-depth analysis of the Aspergillus niger glucoamylase ( $g / a A)$ promoter performance using high-throughput screening and controlled bioreactor cultivation techniques. Biotechnol 2008, 135:266-271.

10. Wiebe MG, Karandikar A, Robson GD, Trinci APJ, Flores Candia JL, Trappe S, Wallis G, Rinas U, Derkx PMF, Madrid SM, Sisniega H, Faus I, Montijn R, Hondel CAMJJ van den, Punt PJ: Production of tissue plasminogen activator (t-PA) in Aspergillus niger. Biotechnol Bioeng 2001, 76:164-174

11. Pel HJ, de Winde JH, Archer DB, Dyer PS, Hofmann G, Schaap PJ, Turner G, de Vries RP, Albang R, Albermann K, Andersen MR, Bendtsen JD, Benen JAE, Berg M van den, Breestraat S, Caddick MX, Contreras R, Cornell M, Coutinho PM, Danchin EGJ, Debets AJM, Dekker P, van Dijck PWM, van Dijk A, Dijkhuizen L, Driessen AJM, D'Enfert C, Geysens S, Goosen C, Groot GSP, et al: Genome sequencing and analysis of the versatile cell factory Aspergillus niger CBS 513.88. Nature Biotechnol 2007, 25:221-231.

12. Jacobs DI, Olsthoorn MMA, Maillet I, Akeroyd M, Breestraat S, Donkers S, Hoeven RAM van der, Hondel CA van den, Kooistra R, Lapointe T, Menke H, Meulenberg R, Misset M, Muller WH, van Peij NNME, Ram A, Rodriguez S, Roelofs MS, Roubos JA, van Tilborg MWEM, Verkleij AJ, Pel HJ, Stam H, Sagt CMJ: Effective lead selection for improved protein production in Aspergillus niger based on integrated genomics. Fungal Genet Biol 2008, 46:S141-S152.

13. Tsang A, Butler G, Powlowsky J, Panisko EA, Baker SE: Analytical and computational approaches to define the Aspergillus secretome. Fungal Genet Biol 2009, 46:S153-S160.

14. Sorensen LM, Lametsch R, Andersen MR, Nielsen PV, Frisvad JC: Proteome analysis of Aspergillus niger : Lactate added in starch-containing medium can increase production of the mycotoxin fumonisin $\mathrm{B} 2$ by modifying acetyl-CoA metabolism. BMC Microbiol 2009, 9:255.

15. Hondmann DHA, Visser J: Carbon metabolism. In Aspergillus: 50 year on Edited by: Martinelli SD, Kinghorn JR. Amsterdam: Elsevier; 1994:61-139.

16. Yuan XL, Kaaij RM van der, Hondel CAMJJ van den, Punt PJ, Maarel MJEC van der, Dijkhuizen L, Ram AFJ: Aspergillus niger genome-wide analysis reveals a large number of novel alpha-glucan acting enzymes with unexpected expression profiles. Mol Genet Genomics 2008, 279:545-561.

17. Vongsangnak W, Salazar M, Hansen K, Nielsen J: Genome-wide analysis of maltose utilization and regulation in aspergilli. Microbiology 2009, 155:3893-3902.

18. Lakshminarayana K, Modi VV, Shah VK: Studies on gluconate metabolism in Aspergillus niger. II. Comparative studies on the enzyme make-up of the adapted and parent strains of Aspergillus niger. Arch Mikrobiol 1969, 66:396-405

19. Muller HM: Utilization of gluconate by Aspergillus niger. I. Enzymes of phosphorylating and nonphosphorylating pathways. Zentralbl Mikrobiol 1985, 140:475-484.

20. te Biesebeke R, Levasseur A, Boussier A, Record E, Hondel CAM van den, Punt PJ: Phylogeny of fungal hemoglobins and expression analysis of the Aspergillus oryzae flavohemoglobin gene fhbA during hyphal growth. Fungal Biol 2009, 114:135-143.

21. Zhou S, Fushinobu S, Nakanishi Y, Kim SW, Wakagi T, Shoun H: Cloning and characterization of two flavohemoglobins from Aspergillus oryzae. Biochem Biophys Res Commun 2009, 381:7-11.

22. Zhao X-J, Raitt D, Burke V, Clewell AS, Kwast KE, Poyton RO: Function and expression of flavohemoglobin in Saccharomyces cerevisiae. Evidence for a role in the oxidative stress response. J Biol Chem 1996, 271:25131-25138.

23. Buisson N, Labbe-Bois R: Flavohemoglobin expression and function in Saccharomyces cerevisiae. No relationship with respiration and complex response to oxidative stress. J Biol Chem 1998, 273:9527-9533.

24. Jami M-S, Barreiro C, Garcia-Estrada C, Martin JF: Proteome analysis of the penicillin producer Penicillium chrysogenum: Characterization of protein changes during the industrial strain improvement. Mol Cell Proteomics 2010 in press.

25. Hasper AA, Visser J, de Graaff LH: The Aspergillus niger transcriptional activator $X \ln R$, which is involved in the degradation of the polysaccharides xylan and cellulose, also regulates D-xylose reductase gene expression. Mol Microbiol 2000, 36:193-200.
26. de Vries RP: Regulation of Aspergillus genes encoding plant cell wall polysaccharide-degrading enzymes; relevance for industrial production. App/ Microbiol Biotechnol 2003, 61:10-20.

27. van Peij NNME, Brinkmann J, Vrsanska M, Visser J, de Graaff LH: $\beta$ Xylosidase activity, encoded by $x \ln D$, is essential for complete hydrolysis of xylan by Aspergillus niger but not for induction of the xylanolytic enzyme spectrum. Eur J Biochem 1997, 245:164-173.

28. van Peij NNME, Gielkens MMC, de Vries RP, Visser J, de Graaff LH: The transcriptional activator $X \ln R$ regulates both xylanolytic and endoglucanase gene expression in Aspergillus niger. Appl Environ Microbiol 1998, 64:3615-3619.

29. Kita K, Matsuzaki K, Hashimoto T, Yanase H, Kato N, Chung MC, Kataoka M, Shimizu S: Cloning of the aldehyde reductase gene from a red yeast, Sporobolomyces salmonicolor, and characterization of the gene and its product. Appl Environ Microbiol 1996, 62:2303-2310.

30. Ford G, Ellis EM: Three aldo-keto reductases of the yeast Saccharomyces cerevisiae. Chem Biol Interact 2001, 130-132:685-698.

31. Cary JW, Bhatnagar D: Nucleotide sequence of a Aspergillus parasiticus gene strongly repressed by thiamine. Biochim Biophys Acta 1995, $1261: 319-320$

32. Manetti AGO, Rosetto M, Maundrell KG: $n$ mt2 of fission yeast: a second thiamine-repressible gene co-ordinately regulated with $n m t 1$. Yeast 1994, 10:1075-1082.

33. Machado CR, Praekelt UM, de Oliveira RC, Barbosa AC, Byrne KL, Meacock PA, Menck CFM: Dual role for the yeast THI4 gene in thiamine biosynthesis and DNA damage tolerance. J Mol Biol 1997, 273:1 14-121.

34. Medina-Silva R, Barros MP, Galhardo RS, Netto LES, Colepicolo P, Menck CFM: Heat stress promotes mitochondrial instability and oxidative responses in yeast deficient in thiazole biosynthesis. Res Microbiol 2006, 157:275-281.

35. Mouyna I, Hartland RP, Fontaine T, Diaquin M, Simenel C, Delepierre M, Henrissat B, Latge JP: A 1,3-beta-glucanosyltransferase isolated from the cell wall of Aspergillus fumigatus is a homologue of the yeast Bgl2p. Microbiology 1998, 144:3171-3180.

36. Lahoz R, Reyes F, Gomez P, Martinez MJ: Lytic enzyme activity in autolysing mycelium of Aspergillus niger. ZAllg Mikrobiol 1983, 23:17-25.

37. Gooday GW, Zhu WY, O'Donnell RW: What are the roles of chitinases in the growing fungus. FEMS Microbio/ Lett 1992, 100:387-392.

38. Yamazaki H, Yamazaki D, Takaya N, Takagi M, Ohta A, Horiuchi H: A chitinase gene, chiB, involved in the autolytic process of Aspergillus nidulans. Curr Genet 2007, 51:89-98.

39. de la Motte RS, Wagner FW: Aspergillus niger sulfhydryl oxidase. Biochemistry 1987, 26:7363-7371.

40. Kawasaki L, Wysong D, Diamond R, Aguirre J: Two divergent catalase genes are differentially regulated during Aspergillus nidulans development and oxidative stress. J Bacteriol 1997, 179:3284-3292

41. Ebina K, Sakagami H, Yokota K, Kondo H: Cloning and nucleotide sequence of CDNA encoding Asp-hemolysin from Aspergillus fumigatus. Biochim Biophys Acta 1994, 1219:148-150.

42. Mattern IE, van Noort JM, Berg P van den, Archer DB, Roberts IN, Hondel CAMJJ van den: Isolation and characterization of mutants of Aspergillus niger deficient in extracellular proteases. Mol Gen Genet 1992, 234:332-336.

43. Punt PJ, Schuren FHJ, Lehmbeck J, Christensen T, Hjort C, Hondel CAMJJ van den: Characterization of the Aspergillus niger prtT, a unique regulator of extracellular protease encoding genes. Fungal Genet Biol 2008, 45:1591-1599.

44. Frederick GD, Rombouts P, Buxton FP: Cloning and characterisation of $p e p C$, a gene encoding a serine protease from Aspergillus niger. Gene 1993, 125:57-64

45. Jarai $\mathrm{G}$, Hombergh $H$ van den, Buxton FP: Cloning and characterization of the pepE gene of Aspergillus niger encoding a new aspartic protease and regulation of pepE and pepC. Gene 1994, 145:171-178.

46. Tenney K, Hunt I, Sweigard J, Pounder JI, McClain C, Bowman EJ, Bowman $B J$ : hex-1, a gene unique to filamentous fungi, encodes the major protein of the Woronin body and functions as a plug for septal pores. Fungal Genet Biol 2000, 31:205-217.

47. Saleeba JA, Cobbett CS, Hynes MJ: Characterization of the amdAregulated aciA gene of Aspergillus nidulans. Mol Gen Genet 1992, 235:349-358. 
48. Chow CM, RajBhandary UL: Developmental regulation of the gene for formate dehydrogenase in Neurospora crassa. J Bacteriol 1993, 175:3703-3709.

49. Tishkov VI, Popov VO: Catalytic mechanism and application of formate dehydrogenase. Biochemistry (Moscow) 2004, 69:1252-1267.

50. Melin P, Schnurer J, Wagner EGH: Proteome analysis of Aspergillus nidulans reveals proteins associated with the response to the antibiotic concanamycin A, produced by Streptomyces species. Mol Genet Genomics 2002, 267:695-702.

51. Asif AR, Oellerich M, Amstrong VW, Riemenschneider B, Monod M, Reichard U: Proteome of conidial surface associated proteins of Aspergillus fumigatus reflecting potential vaccine candidates and allergens. J Proteome Res 2006, 5:954-962.

52. Ng AML, Smith JE, McIntosh AF: Influence of dilution rate on enzyme synthesis in Aspergillus niger continuous culture. J Gen Microbiol 1974, 81:425-434.

53. Jorgensen TR, Goosen T, Hondel CA, Ram AFJ, Iversen JJ: Transcriptomic comparison of Aspergillus niger growing on two different sugars reveals coordinated regulation of the secretory pathway. $B M C$ Genomics 2009, 10:44.

54. El-Enshasy H, Hellmuth K, Rinas U: GpdA -promoter controlled production of glucose oxidase by recombinant Aspergillus niger using nonglucose carbon sources. Appl Biochem Biotechnol 2001, 90:57-66.

55. Hellmuth K, Pluschkell S, Jung JK, Ruttkowski E, Rinas U: Optimization of glucose oxidase production by Aspergillus niger using genetic- and process-engineering techniques. Appl Microbiol Biotechnol 1995, 43:978-984.

56. El-Enshasy $\mathrm{H}$, Hellmuth $\mathrm{K}$, Rinas U: Fungal morphology in submerged cultures and its relation to glucose oxidase excretion by recombinant Aspergillus niger. Appl Biochem Biotechnol 1999, 81:1-11.

57. van Hartingsveldt W, Mattern IE, van Zeijl CMJ, Pouwels PH, Hondel CAMJJ van den: Development of a homologous transformation system for Aspergillus niger based on the pyrG gene. Mol Gen Genet 1987 206:71-75.

58. Bos CJ, Debets AJM, Swart K, Huybers A, Kobus G, Slakhorst SM: Genetic analysis and the construction of master strains for assignment of genes to six linkage groups in Aspergillus niger. Curr Genet 1988, 14:437-443.

59. Vogel HJ: A convenient growth medium for Neurospora (Medium N). Microbial Genet Bull 1956, 243:112-119.

60. Bradford MM: A rapid and sensitive method for the quantitation of microgram quantities of protein utilizing the principle of protein-dyebinding. Anal Biochem 1976, 72:248-254.

61. Candiano G, Bruschi M, Musante L, Santucci L, Ghiggeri GM, Carnemolla B, Orecchia P, Zardi L, Righetti PG: Blue silver: a very sensitive colloidal Coomassie G-250 staining for proteome analysis. Electrophoresis 2004, 25:1327-1333.

62. Wang W, Sun J, Nimtz M, Deckwer W-D, Zeng A-P: Protein identification from two-dimensional gel electrophoresis analysis of Klebsiella pneumoniae by combined use of mass spectrometry data and raw genome sequences. Proteome Sci 2003, 1:6.

63. Wissing J, Godl K, Brehmer D, Blencke S, Weber M, Habenberger P, SteinGerlach M, Missio A, Cotten M, Muller S, Daub H: Chemical proteomic analysis reveals alternative modes of action for pyrido[2,3d]pyrimidine kinase inhibitors. Mol Cell Proteomics 2004, 3:1181-1193.

64. Sun J, Lu X, Rinas U, Zeng AP: Metabolic peculiarities of Aspergillus niger disclosed by comparative metabolic genomics. Genome Biol 2007, 8:R182.

doi: $10.1186 / 1475-2859-9-23$

Cite this article as: Lu et al., The intra- and extracellular proteome of Aspergillus niger growing on defined medium with xylose or maltose as carbon substrate Microbial Cell Factories 2010,9:23

\section{Submit your next manuscript to BioMed Central} and take full advantage of:

- Convenient online submission

- Thorough peer review

- No space constraints or color figure charges

- Immediate publication on acceptance

- Inclusion in PubMed, CAS, Scopus and Google Scholar

- Research which is freely available for redistribution

Submit your manuscript at www.biomedcentral.com/submit
C BioMed Central 